Energy Technology Division Energy Technology Division Energy Technology Division Energy Technology Division Energy Technology Division Energy Technology Division Energy Technology Division Energy Technology Division Energy Technology Division Energy Technology Division Energy Technology Division Energy Technology Division Energy Technology Division Energy Technology Division Energy Technology Division Energy Technology Division Energy Technology Division Energy Technology Division Energy Technology Division Energy Technology Division Energy Technology Division Energy Technology Division Energy Technology Division Energy Technology Division Energy Technology Division Energy Technology Division Energy Technology Division Energy Technology Division Energy Technology Division Energy Technology Division Energy Technology Division Energy Technology Division Energy Technology Division Energy Technology Division

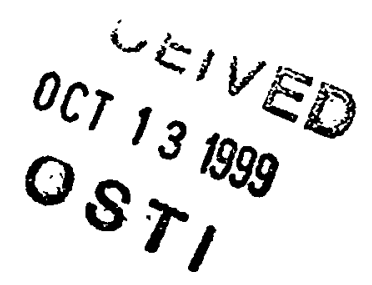

ANL-97/16

\title{
Large Eddy Simulation and Its Implementation in the COMMIX Code
}

by Da-Hai Yu and Jiangang Sun

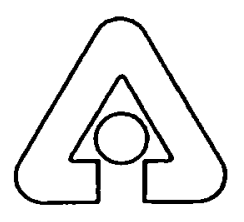

Argonne National Laboratory, Argonne, Illinois 60439

operated by The University of Chicago

for the United States Department of Energy under Contract W-31-109-Eng-38

Energy Technology Division

Energy Technology Division

Energy Technology Division

Energy Technology Division

Energy Technology Division

Energy Technology Division

Energy Technology Division

Energy Technology Division 
Argonne National Laboratory, with facilities in the states of Illinois and Idaho, is owned by the United States government, and operated by The University of Chicago under the provisions of a contract with the Department of Energy.

\section{DISCLAIMER}

This report was prepared as an account of work sponsored by an agency of the United States Government. Neither the United States Government nor any agency thereof, nor any of their employees, makes any warranty, express or implied, or assumes any legal liability or responsibility for the accuracy, completeness, or usefulness of any information, apparatus, product, or process disclosed, or represents that its use would not infringe privately owned rights. Reference herein to any specific commercial product, process, or service by trade name, trademark, manufacturer, or otherwise, does not necessarily constitute or imply its endorsement, recommendation, or favoring by the United States Government or any agency thereof. The views and opinions of authors expressed herein do not necessarily state or reflect those of the United States Government or any agency thereof.

Reproduced from the best available copy.

Available to DOE and DOE contractors from the Office of Scientific and Technical Information

P.O. Box 62

Oak Ridge, TN 37831

Prices available from (423) 576-8401

Available to the public from the National Technical Information Service

U.S. Department of Commerce 5285 Port Royal Road Springfield, VA 22161 


\section{Large Eddy Simulation and Its Implementation in the COMMIX Code}

by

Da-Hai Yu* and Jiangang Sun

Energy Technology Division

*Department of Applied Mathematics and Statistics State University of New York at Stony Brook

Stony Brook, NY 11794-3600

November 1998

Work sponsored by

U. S. Nuclear Regulatory Commission Office of Nuclear Regulatory Research

Division of System Research

and by

Argonne National Laboratory 


\section{DISCLAIMER}

Portions of this document may be illegible in electronic image products. Images are produced from the best available original document. 


\section{Contents}

$\begin{array}{ll}\text { Abstract } & 1\end{array}$

1 Introduction 2

2 The Large Eddy Simulation Method 3

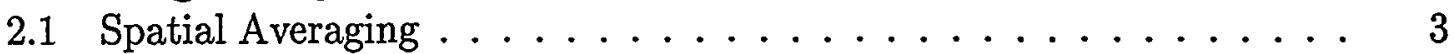

2.2 Subgrid Scale Model . . . . . . . . . . . . . . . 3

2.3 Governing Equations of LES Method .............. . . . . 5

2.4 Dynamic LES . . . . . . . . . . . . . . . . . 5

2.5 The COMMIX Code and Implementation of

LES in COMMIX ................... 7

3 Simulation of Flow over Square Prism

$\begin{array}{ll}\text { with LES } & 7\end{array}$

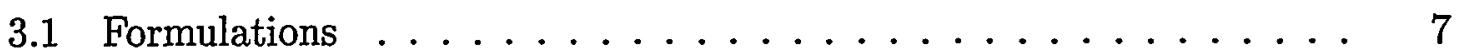

3.2 Flow Geometry ................................... 10

3.3 Simulation Results of Velocity Field . . . . . . . . . . 10

3.4 Simulation Results of Pressure Field . . . . . . . . . . . 12

3.4.1 Mean Pressure Distribution on Surface of Prism . . . . . . 12

3.4.2 RMS Pressure Distribution on Surface of Prism . . . . . . 13

3.4 .3 Integral Quantities ................ 13

3.5 2-D Simulation Results from COMMIX . . . . . . . . . 15

4 Summary 16

$\begin{array}{ll}\text { Acknowledgments } & 17\end{array}$

$\begin{array}{ll}\text { References } & 17\end{array}$ 


\section{Figures}

1 Schematic representation of flow geometry. . . . . . . . . . 9

2 Time-averaged streamwise velocity. . . . . . . . . . . . . 10

3 Time history of $w$-component velocity in wake. . . . . . . . . 11

4 Distribution of mean pressure coefficient on surface of square prism . 12

5 Distribution of RMS pressure. . . . . . . . . . . . . 13

6 Chordwise correlation of pressure. . . . . . . . . . . . . 14

7 COMMIX results of time-averaged streamwise velocity. . . . . . 15

8 COMMIX results of distribution of mean pressure coefficient along

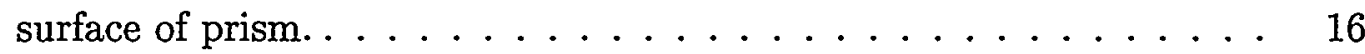

\section{Tables}

1 Calculated and experimental integral quantities. . . . . . . . 14 


\title{
LARGE EDDY SIMULATION AND ITS IMPLEMENTATION IN THE COMMIX CODE \\ by \\ Dahai Yu and Jiangang Sun
}

\begin{abstract}
Large eddy simulation (LES) is a numerical simulation method for turbulent flows and is derived by spatial averaging of the Navier-Stokes equations. In contrast with the Reynolds-averaged Navier-Stokes equations (RANS) method, LES is capable of calculating transient turbulent flows with greater accuracy. Application of LES to differing flows has given very encouraging results, as reported in the literature. In recent years, a dynamic LES model that presented even better results was proposed and applied to several flows. This report reviews the LES method and its implementation in the COMMIX code, which was developed at Argonne National Laboratory. As an example of the application of LES, the flow around a square prism is simulated, and some numerical results are presented. These results include a three-dimensional simulation that uses a code developed by one of the authors at the University of Notre Dame, and a two-dimensional simulation that uses the COMMIX code. The numerical results are compared with experimental data from the literature and are found to be in very good agreement.
\end{abstract}

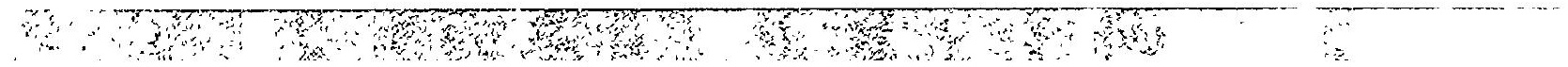




\section{Introduction}

Numerical simulations of fluid flows are being used more and more widely in many disciplines, e.g., meteorology, aeronautics, heat transfer, and civil engineering. This increasing use has become feasible both by the availability of ever-improving fast computers and by the development of computational fluid dynamics.

The most straightforward way to simulate a fluid flow is direct numerical simulation (DNS), so designated because no turbulence model is required. The feasibility of DNS is limited to low-Reynolds-number flows, because the number of mesh points for a DNS is proportional to $R e^{9 / 4}$, which makes it impossible for current or near-future computers to simulate high-Reynolds-number flows. To overcome this difficulty, many investigators have developed differing methods of treating the turbulence effect. Most of these methods fall into two categories, namely Reynolds-averaged Navier-Stokes (RANS) equations and large eddy simulation (LES).

Compared with the LES method, the RANS method has a longer history of application, and a lower computational cost. Much experience has been accumulated with this method, especially with the $k-\epsilon$ version of the method. Despite its achievements, RANS is not sufficiently accurate for separating and reattaching flows. Review papers about this method have been published (Launder and Spalding, 1972; Arpaci and Larsen, 1974); hence, the method will not be discussed in detail in this report.

Instead of using ensemble- or time-averaging, as in RANS, LES uses spatial averaging and only simulates large-scale motions explicitly, while leaving small-scale eddies for modeling. Such an approach to reproduce the turbulence effect is based on two experimental observations. One is that large-scale eddies in a turbulent flow depend on the configuration of the flow, are anisotropic, and contain most of the energy. The other observation is that small-scale eddies in a turbulent flow are more independent of the flow, are isotropic, and contain a small part of the total energy. These observations suggest the possibility of modeling the small-scale eddies while explicitly computing the large-scale flow, as conducted in the LES.

The LES method is implemented in the COMMIX code, which was developed at Argonne National Laboratory (ANL). Several turbulence models, including the constant-viscosity model and the $k-\epsilon$ model, have already been incorporated into COMMIX. After implementation of the LES in the COMMIX code, the application of COMMIX may be expanded to additional flows.

As a example of the application of LES, we present some numerical results obtained at ANL and the University of Notre Dame on flow around a square prism. The numerical results are compared with experimental data and other numerical results, and demonstrate good agreement with experimental observations and thus confirm the accuracy of the LES. 


\section{The Large Eddy Simulation Method}

\subsection{Spatial Averaging}

The LES method uses short-range spatial averaging of the Navier-Stokes equations. This averaging operation to any quantity, e.g., $u$, can be written as

$$
\bar{u}(\mathrm{x})=\int u\left(\mathrm{x}^{\prime}\right) \bar{G}\left(\mathrm{x}, \mathrm{x}^{\prime} ; \bar{\Delta}_{f}\right) d \mathrm{x}^{\prime},
$$

where $\bar{G}$ is a filter weighting function, $\bar{\Delta}_{f}$ is the associated length scale of the filter, $\mathbf{x}$ represents the space coordinates, and $\mathbf{x}^{\prime}$ is a dummy variable for the integral. The most frequently used filter functions are the top-hat function in physical space, and the sharp spectral cut function in wavenumber space (Mason, 1989). Averaging of the Navier-Stokes equations removes the scales that are smaller than $\bar{\Delta}_{f}$.

A quantity can be divided into the sum of its average value and its fluctuation, i.e.,

$$
u_{i}=\bar{u}_{i}+u_{i}^{\prime} .
$$

It can be understood that the averaged values $\left(\bar{u}_{i}\right)$ contain the large-scale motions, while the fluctuation term $\left(u_{i}^{\prime}\right)$ represents motions related to the small-scales. These fluctuations will no longer appear in the averaged Navier-Stokes equations.

The average of the nonlinear term in the Navier-Stokes equations related to convection is

$$
\overline{u_{i} u_{j}}=\overline{\bar{u}_{i} \bar{u}_{j}}+\overline{\bar{u}_{i} u_{j}^{\prime}}+\overline{u_{i}^{\prime} \bar{u}_{j}}+\overline{u_{i}^{\prime} u_{j}^{\prime}} .
$$

In Reynolds averaging, the second and third terms can be eliminated, and the second averaging (upper bar) of the first term can be dropped. For spatial averaging, neither of these can be done. The term to be modeled can be expressed as

$$
\begin{aligned}
\tau_{i j}^{\prime} & =\overline{u_{i} u_{j}}-\bar{u}_{i} \bar{u}_{j} \\
& =\overline{\bar{u}_{i} \bar{u}_{j}}-\bar{u}_{i} \bar{u}_{j}+\overline{\overline{u_{i} u_{j}^{\prime}}}+\overline{u_{i}^{\prime} \bar{u}_{j}}+\overline{u_{i}^{\prime} u_{j}^{\prime}} .
\end{aligned}
$$

It is the role of a turbulence model to relate $\tau_{i j}^{\prime}$ to the spatial averaged velocity $\bar{u}_{i}$. Such a model is often called a subgrid scale model. Theoretically, it is more proper to call $\tau_{i j}^{\prime}$ a subfilter scale model, because the filter scale is not necessarily the same as the computational grid size. In reality, it is often recommended that the filter scale be larger than the grid size.

\subsection{Subgrid Scale Model}

The most widely used subgrid scale model was first proposed by Smagorinsky (1963) and is usually named after him. In LES, the scales to be modeled are small enough to be within the inertial subrange, and can therefore be considered isotropic. In such a case, it is possible to introduce a scalar viscosity to relate the subgrid stress to the large-scale (or averaged) velocity strain rate. Then,

$$
\tau_{i j}^{\prime}=-\nu_{t}\left(\frac{\partial \bar{u}_{i}}{\partial x_{j}}+\frac{\partial \bar{u}_{j}}{\partial x_{i}}\right)
$$


where $\nu_{t}$ is an eddy viscosity and has dimensions $L L / T$. A natural choice of length scale is $\bar{\Delta}_{f}$, and the velocity scale is the product of $\bar{\Delta}_{f}$ and the strain rate $S$, i.e.,

$$
L=\bar{\Delta}_{f}
$$

and

$$
L / T=\bar{\Delta}_{f} \cdot S,
$$

where

$$
S^{2}=\frac{1}{2}\left(\frac{\partial \bar{u}_{i}}{\partial x_{j}}+\frac{\partial \bar{u}_{j}}{\partial x_{i}}\right)\left(\frac{\partial \bar{u}_{i}}{\partial x_{j}}+\frac{\partial \bar{u}_{j}}{\partial x_{i}}\right) .
$$

Thus,

$$
\nu_{t}=\left(C_{s} \bar{\Delta}_{f}\right)^{2} S,
$$

where $C_{s}$ is a constant. It is clear from this expression that the eddy viscosity is the product of the local shear and a length scale. This model is a three-dimensional (3-D) application of mixing-length turbulence modeling.

These assumptions lead to the Smagorinsky model for subgrid-scale stress

$$
\tau_{i j}^{\prime}=-\left(C_{s} \bar{\Delta}_{f}\right)^{2} S\left(\frac{\partial \bar{u}_{i}}{\partial x_{j}}+\frac{\partial \bar{u}_{j}}{\partial x_{i}}\right),
$$

which will be used in the Navier-Stokes equations.

The value of $C_{s}$ depends on the nature of the filter and can be determined theoretically for some mathematically defined filters. If the filter operation is in the range of the inertial subrange, the energy spectrum is

$$
E=\alpha \epsilon^{2 / 3} k^{-5 / 3},
$$

where $\alpha$ is a constant, experimentally determined as $1.5 ; \epsilon$ is the dissipation; and $k$ is the wave number of the spectrum. For a sharp-spectral-cut filter with a wave number of $\pi / \bar{\Delta}_{f}$, the total subfilter scale energy is

$$
E=\int_{\pi / \bar{\Delta}_{f}}^{\infty} E d k=\frac{3}{2} \alpha \epsilon^{2 / 3}\left(\frac{\bar{\Delta}_{f}}{\pi}\right)^{2 / 3},
$$

and the resolved shear is

$$
S^{2}=2 \int_{\infty}^{\pi / \bar{\Delta}_{f}} k^{2} E d k=\frac{3}{2} \alpha \epsilon^{2 / 3}\left(\frac{\bar{\Delta}_{f}}{\pi}\right)^{-4 / 3} .
$$

Lilly (1967) noted that with the Smagorinsky model,

$$
\epsilon=\left(C_{s} \bar{\Delta}_{f}\right)^{2} S^{3} .
$$

Substituting Eq. 15 into Eq. 14, we obtain

$$
C_{s}=\frac{1}{\pi}\left(\frac{3 \alpha}{2}\right)^{-3 / 4} .
$$

From the usually quoted value of $\alpha=1.5$, we can determine that $C_{s}=0.17$. In practical use, the value of $C_{s}$ is between 0.1 and 0.2 . 


\subsection{Governing Equations of LES Method}

The 3-D unsteady Navier-Stokes equation for incompressible flows can be written in nondimensional form as

$$
\begin{aligned}
\frac{\partial u_{i}}{\partial t}+\frac{\partial u_{i} u_{j}}{\partial x_{j}} & =-\frac{\partial P}{\partial x_{i}}+\frac{1}{R e} \frac{\partial^{2} u_{i}}{\partial x_{j} \partial x_{j}} \\
\frac{\partial u_{i}}{\partial x_{i}} & =0 .
\end{aligned}
$$

Taking the spatial averaging as specified in Eq. 1, the averaged equations are,

$$
\begin{aligned}
\frac{\partial \bar{u}_{i}}{\partial t}+\frac{\partial \overline{u_{i} u_{j}}}{\partial x_{j}} & =-\frac{\partial \bar{P}}{\partial x_{i}}+\frac{1}{R e} \frac{\partial^{2} \bar{u}_{i}}{\partial x_{j} \partial x_{j}} \\
\frac{\partial \bar{u}_{i}}{\partial x_{i}} & =0 .
\end{aligned}
$$

From Eq. 4, the convection term can be written as

$$
\frac{\partial \overline{u_{i} u_{j}}}{\partial x_{j}}=\frac{\partial \bar{u}_{i} \bar{u}_{j}}{\partial x_{j}}+\frac{\partial \tau_{i j}^{\prime}}{\partial x_{j}}
$$

By combining Eqs. 19 and 21, we can express the momentum equation for the LES as

$$
\frac{\partial \bar{u}_{i}}{\partial t}+\frac{\partial \bar{u}_{i} \bar{u}_{j}}{\partial x_{j}}=-\frac{\partial \bar{P}}{\partial x_{i}}+\frac{1}{R e} \frac{\partial^{2} \bar{u}_{i}}{\partial x_{j} \partial x_{j}}-\frac{\partial \tau_{i j}^{\prime}}{\partial x_{j}}
$$

(Pinelli, 1989; Murakami et al., 1995), where $\tau_{i j}^{\prime}$ can be computed from either the Smagorinsky model, which is expressed in Eq. 11, or by other models. Thus, a complete set of LES equations would include the continuity Eq. 20, the momentum Eq. 22, and a subgrid scale model, such as the Smagorinsky model in Eq. 11.

\subsection{Dynamic LES}

Over the years, much effort has been devoted to developing new subgrid scale models and examining the performance of existing models. Some of these developments are reviewed by Boris et al. (1992) and Lesieur and Metais (1996). Here, we present a short description of the dynamic LES model, which was proposed by Germano et al. (1991), and is an encouraging improvement over the conventional Smagorinsky model. The basic purpose of the dynamic LES model is to compute the value of $C_{s}$ from instantaneous flow instead of specifying its value as a constant.

Besides the original filter $\bar{G}$ defined in Eq. 1 , a test filter $\tilde{G}$ in the dynamic LES is defined as

$$
\tilde{u}(\mathbf{x})=\int u\left(\mathbf{x}^{\prime}\right) \tilde{G}\left(\mathbf{x}, \mathbf{x}^{\prime} ; \tilde{\Delta}_{f}\right) d \mathbf{x}^{\prime}
$$

The test filter is assumed to be wider than filter $\bar{G}$. Another filter is defined as $\tilde{G}=\tilde{G} \bar{G}$. Application of filter $\bar{G}$ to the Navier-Stokes equations gives Eqs. 20 and 
22. Similarly, application of filter $\tilde{\bar{G}}$ to the equations of motion gives

$$
\frac{\partial \tilde{\bar{u}}_{i}}{\partial t}+\frac{\partial \tilde{\bar{u}}_{i} \tilde{\bar{u}}_{j}}{\partial x_{j}}=-\frac{\partial \tilde{\bar{P}}}{\partial x_{i}}+\frac{1}{R e} \frac{\partial^{2} \tilde{\bar{u}}_{i}}{\partial x_{j} \partial x_{j}}-\frac{\partial T_{i j}}{\partial x_{j}}
$$

where the subgrid-scale stress is now

$$
T_{i j}=\widetilde{u_{i} u_{j}}-\tilde{\bar{u}}_{i} \tilde{\bar{u}}_{j} .
$$

Also, the resolved turbulence stress $\mathcal{T}$ is defined as

$$
\mathcal{T}_{i j}=\widetilde{\bar{u}}_{i} \bar{u}_{j}-\tilde{\bar{u}}_{i} \tilde{\bar{u}}_{j} .
$$

The resolved stresses represent the contribution to the Reynolds stresses of the scales whose length is intermediate between the filter width $\bar{\Delta}_{f}$ of filter $\bar{G}$, and the test filter width, i.e., that of filter $\tilde{\bar{G}}$. The relationship of the quantities given in Eqs. 4, 25, and 26 is

$$
\mathcal{T}_{i j}=T_{i j}-\tilde{\tau}_{i j}
$$

In Eq. 27, the resolved turbulence stress $\mathcal{T}_{i j}$ can be calculated explicitly, whereas the subgrid-scale stresses at the test and original levels $T_{i j}$ and $\tau_{i j}$ may be modeled. Thus, Eq. 27 can be used to derive more accurate subgrid-scale stress models by determining the value of the Smagorinsky coefficient most appropriate to the instantaneous state of the flow. Assuming that the Smagorinsky model can be used for both $T_{i j}$ and $\tau_{i j}$, and allowing $M_{i j}$ and $m_{i j}$ to be the models for the anisotropic parts of $T_{i j}$ and $\tau_{i j}$,

$$
\tau_{i j}-\left(\delta_{i j} / 3\right) \tau_{k k} \simeq m_{i j}=-2 C_{s} \bar{\Delta}^{2}\|\bar{S}\| \bar{S}_{i j}
$$

and

$$
T_{i j}-\left(\delta_{i j} / 3\right) T_{k k} \simeq M_{i j}=-2 C_{s} \tilde{\bar{\Delta}}^{2} \| \tilde{\bar{S}}_{\|} \tilde{\bar{S}}_{i j}
$$

where

$$
\tilde{\bar{S}}_{i j}=\frac{1}{2}\left(\frac{\partial \tilde{\bar{u}}_{i}}{\partial x_{j}}+\frac{\partial \tilde{\bar{u}}_{j}}{\partial x_{i}}\right), \quad\|\widetilde{\widetilde{S}}\|=\sqrt{2} \widetilde{\bar{u}}_{m n} \tilde{\bar{u}}_{m n},
$$

$\bar{\Delta}$ is the characteristic filter width associated with $\bar{G}$, and $\tilde{\bar{\Delta}}$ is the filter width associated with $\widetilde{\bar{G}}$. Substituting Eqs. 28 and 29 into Eq. 27 and contracting with $\bar{S}_{i j}$, we obtain

$$
\mathcal{T}_{i j} \bar{S}_{i j}=-2 C_{s}\left(\tilde{\bar{\Delta}}^{2}\left\|\tilde{\bar{S}}_{\|} \tilde{\bar{S}}_{i j} \bar{S}_{i j}-\bar{\Delta}^{2}\right\| \tilde{\bar{S}}^{\|} \tilde{\bar{S}}_{i j} \bar{S}_{i j}\right),
$$

from which we can theoretically obtain $C_{s}(x, y, z, t)$. These are the basic principles of the dynamic LES model. Details about this model are available in the original paper by Germano et al. (1991). Applications of the model to various flows have given very encouraging results. 


\subsection{The COMMIX Code and Implementation of LES in COMMIX}

The COMMIX (COMponent MIXing) code was developed at ANL for analysis of single-phase multicomponent fluid flow and heat transfer problems. It uses a finitevolume method, in which staggered grids divide the space domain and primary variable Navier-Stokes equations are solved. Several options are available for convection term discretization, including upwind schemes in COMMIX-1C and central-difference and QUICK schemes in an in-house version. The viscous terms are discretized by a second-order central-difference method. A semi-implicit algorithm, derived from the Los Alamos ICE Technique, is used for temporal advance. Details of the COMMIX code are given in the manual by Domanus et al. (1990).

The original COMMIX contained two turbulence models; one, a constant-turbulence-viscosity model, the other, a $k-\epsilon$ two-equation turbulence model. Now, we have implemented the LES method in COMMTX. The value used in the code for parameter $C_{s}$ is 0.15 , which is mid-range of $0.1-0.2$, the most widely used values. This work was conducted from June 20 to September 20, 1995, in the Energy Technology Division of ANL. The code was compiled and debugged, and a test two-dimensional (2-D) computation of flow over a square prism was conducted. Some representative results from this 2-D simulation are presented in Section 3.

As an example of the application of LES, in the next section, we present results obtained when this technique was used for a 3-D simulation of flow over the square prism. These results were obtained at the University of Notre Dame with a code, independent of the COMMIX, that was written by one of the authors.

\section{Simulation of Flow over Square Prism with LES}

\subsection{Formulations}

The governing equations for the flow are Eqs. 11, 20, and 22. These equations are nondimensionalized by using the length of the front side of the rectangular cross section $L$ and the inflow velocity $U_{0}$. Time is nondimensionalized by $L / U_{0}$ and pressure, by $\rho U_{0}^{2}$, where $\rho$ is the mass density of the fluid. Thus, the Reynolds number is defined as $L U_{0} / \nu$, where $\nu$ is the fluid kinematic viscosity.

The boundary conditions on the solid walls are of the no-slip, no-penetration type, i.e., all of the velocity components of the flow in the $x_{1}, x_{2}$, and $x_{3}$ directions are set to zero on the surface. To be consistent with the accuracy of the numerical scheme, imaginary points inside the solid wall boundaries are specified with a quadratic interpolation. The inflow boundary condition is a constant-uniform-velocity set equal to unity in the $x_{1}$ direction and zero in the $x_{2}$ and $x_{3}$ directions. The upper and lower sides of the flow domain are set with the condition $\partial / \partial n=0$. For the outflow boundary, a similar free-boundary condition $\partial / \partial n=0$ can be used at infinity. However, because the numerical simulation must be conducted in a finite domain, a special 
treatment of this condition is required. In this simulation, we used the convective outflow boundary condition

$$
\frac{\partial \bar{u}_{i}}{\partial t}+U_{0} \frac{\partial \bar{u}_{i}}{\partial x_{1}}=0, \quad i=1,2,3 .
$$

Simulation results confirm this boundary condition to be both stable and accurate for a Reynolds number of $10^{5}$.

In this simulation, the computational domain is discretized with a staggered grid, as in the marker and cell (MAC) method proposed by Harlow and Welch (1965). In studies that involve a subgrid-scale model of turbulence viscosity, it is important that the accuracy order of the finite-difference scheme be high enough to ensure that the numerical diffusion caused by the discretization does not dwarf the turbulence viscosity. Such being the case, a third-order upwind finite-difference scheme for the convection terms (Leonard, 1979; Davis and Moore, 1982, 1984), and a Leith-type scheme for the temporal marching, were applied in the simulation conducted by $\mathrm{Yu}$ and Kareem (1996) at Notre Dame. The discretization algorithm has been integrated with the LES model so the characteristics of high-Reynolds-number flows are captured. The central-difference and QUICK schemes (Leonard, 1979) can be expressed in one equation,

$$
\begin{aligned}
\frac{\partial \phi}{\partial x} \simeq & \frac{1}{D x}\left\{\left[\frac{1}{2}\left(\phi_{i}+\phi_{i+1}\right)-\frac{q}{8}\left(\phi_{i-1}-2 \phi_{i}+\phi_{i+1}\right)\right]\right. \\
- & {\left.\left[\frac{1}{2}\left(\phi_{i-1}+\phi_{i}\right)-\frac{q}{8}\left(\phi_{i-2}-2 \phi_{i-1}+\phi_{i}\right)\right]\right\}, }
\end{aligned}
$$

where $q=1$ corresponds to the QUICK scheme, and $q=0$ corresponds to the central-difference scheme. Detailed derivations of the scheme are omitted for brevity. Interested readers are referred to the paper by Yu and Kareem (1996). The discretized $3-\mathrm{D}$ equation is given by

$$
\begin{aligned}
\phi_{P}^{N+1}= & \phi_{P}^{N}+\left\{-C_{E}\left[\frac{1}{2}\left(\phi_{P}+\phi_{E}\right)-\frac{1}{2} C_{E}\left(\phi_{E}-\phi_{P}\right)\right.\right. \\
& \left.-\left(\frac{q}{8}+\frac{1}{24}-\gamma_{1}-\frac{1}{6} C_{E}^{2}\right)\left(\phi_{E}-2 \phi_{P}+\phi_{W}\right)\right] \\
& +C_{W}\left[\frac{1}{2}\left(\phi_{P}+\phi_{W}\right)-\frac{1}{2} C_{W}\left(\phi_{P}-\phi_{W}\right)\right. \\
& \left.-\left(\frac{q}{8}+\frac{1}{24}-\gamma_{1}-\frac{1}{6} C_{W}^{2}\right)\left(\phi_{W W}-2 \phi_{W}+\phi_{P}\right)\right] \\
& -C_{N}\left[\frac{1}{2}\left(\phi_{P}+\phi_{N}\right)-\frac{1}{2} C_{N}\left(\phi_{N}-\phi_{P}\right)\right. \\
& \left.-\left(\frac{q}{8}+\frac{1}{24}-\gamma_{2}-\frac{1}{6} C_{N}^{2}\right)\left(\phi_{N}-2 \phi_{P}+\phi_{S}\right)\right] \\
& +C_{S}\left[\frac{1}{2}\left(\phi_{P}+\phi_{S}\right)-\frac{1}{2} C_{S}\left(\phi_{P}-\phi_{S}\right)\right. \\
& \left.-\left(\frac{q}{8}+\frac{1}{24}-\gamma_{2}-\frac{1}{6} C_{S}^{2}\right)\left(\phi_{P}-2 \phi_{S}+\phi_{S S}\right)\right]
\end{aligned}
$$




$$
\begin{aligned}
& -C_{F}\left[\frac{1}{2}\left(\phi_{P}+\phi_{F}\right)-\frac{1}{2} C_{F}\left(\phi_{F}-\phi_{P}\right)\right. \\
& \left.-\left(\frac{q}{8}+\frac{1}{24}-\gamma_{3}-\frac{1}{6} C_{F}^{2}\right)\left(\phi_{F}-2 \phi_{P}+\phi_{B}\right)\right] \\
& +C_{B}\left[\frac{1}{2}\left(\phi_{P}+\phi_{B}\right)-\frac{1}{2} C_{B}\left(\phi_{P}-\phi_{B}\right)\right. \\
& \left.-\left(\frac{q}{8}+\frac{1}{24}-\gamma_{3}-\frac{1}{6} C_{B}^{2}\right)\left(\phi_{P}-2 \phi_{B}+\phi_{B B}\right)\right] \\
& +\gamma_{1}\left(\phi_{E}-2 \phi_{P}+\phi_{W}\right)+\gamma_{2}\left(\phi_{N}-2 \phi_{P}+\phi_{S}\right) \\
& \left.+\gamma_{3}\left(\phi_{F}-2 \phi_{P}+\phi_{B}\right)+s_{p} D t\right\}^{N}
\end{aligned}
$$

where $P$ denotes the present point, $W$ denotes the point west (to the left) of the present point in the $x_{1}$ direction, and $W W$ denotes the point farther to the west. The subscripts $E$ (east), $N$ (north), $S$ (south), $F$ (front) and $B$ (back) may be interpreted in a similar fashion. $C_{E}$ is the Courant number on the east side of the present point, i.e., $C_{E}=u_{E} \cdot D t / D x_{1} \cdot \gamma_{1}=\left(\nu+\nu_{t}\right) D t / D x_{1}{ }^{2}, \gamma_{2}=\left(\nu+\nu_{t}\right) D t / D x_{2}{ }^{2}$, and $\gamma_{3}=\left(\nu+\nu_{t}\right) D t / D x_{3}{ }^{2}$. Here $\nu_{t}$ is the subgrid-scale viscosity, calculated with Eq. 10. The pressure field is solved with a successive overrelaxation method, ensuring that the computed flow field is free of divergence. The equations presented here are for a uniform grid mesh, but both uniform and nonuniform grid meshes were used in the calculations.

For the simulations with the COMMIX code, we used the built-in discretization methods, which deployed the central-difference method for both the convection and viscous terms. The pressure equation and the marching in time were also solved with built-in methods.

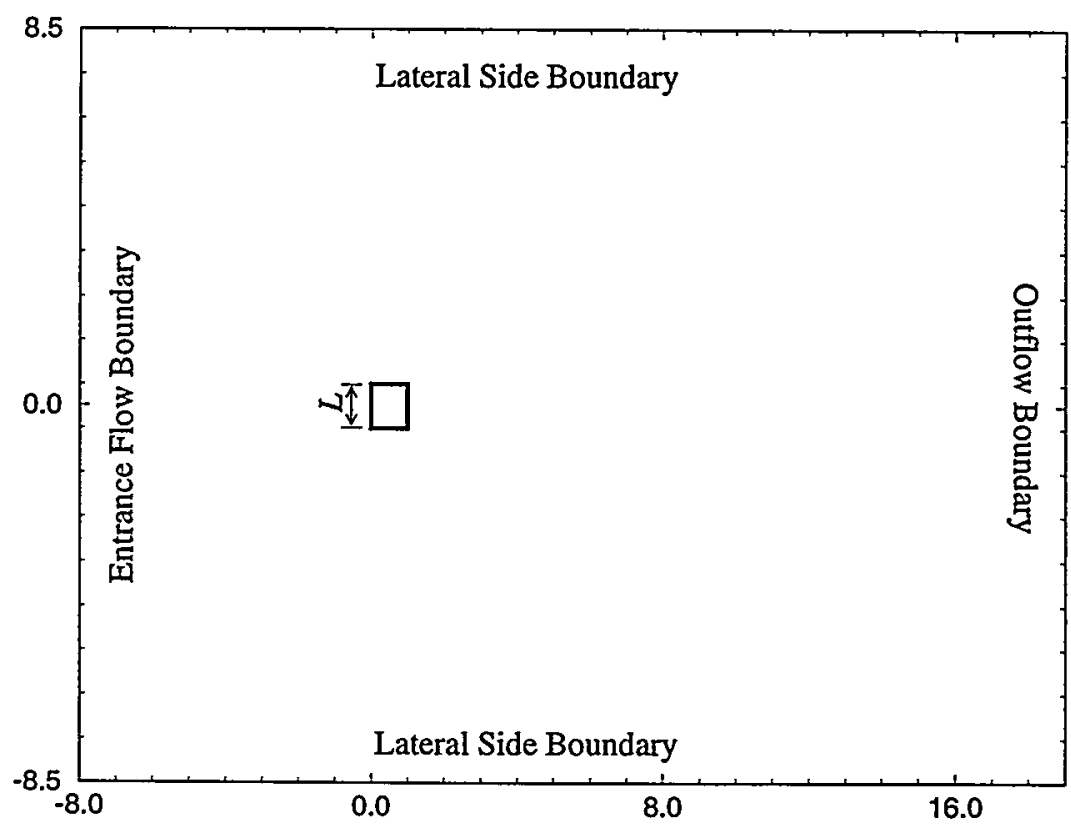

Figure 1. Schematic representation of flow geometry. 


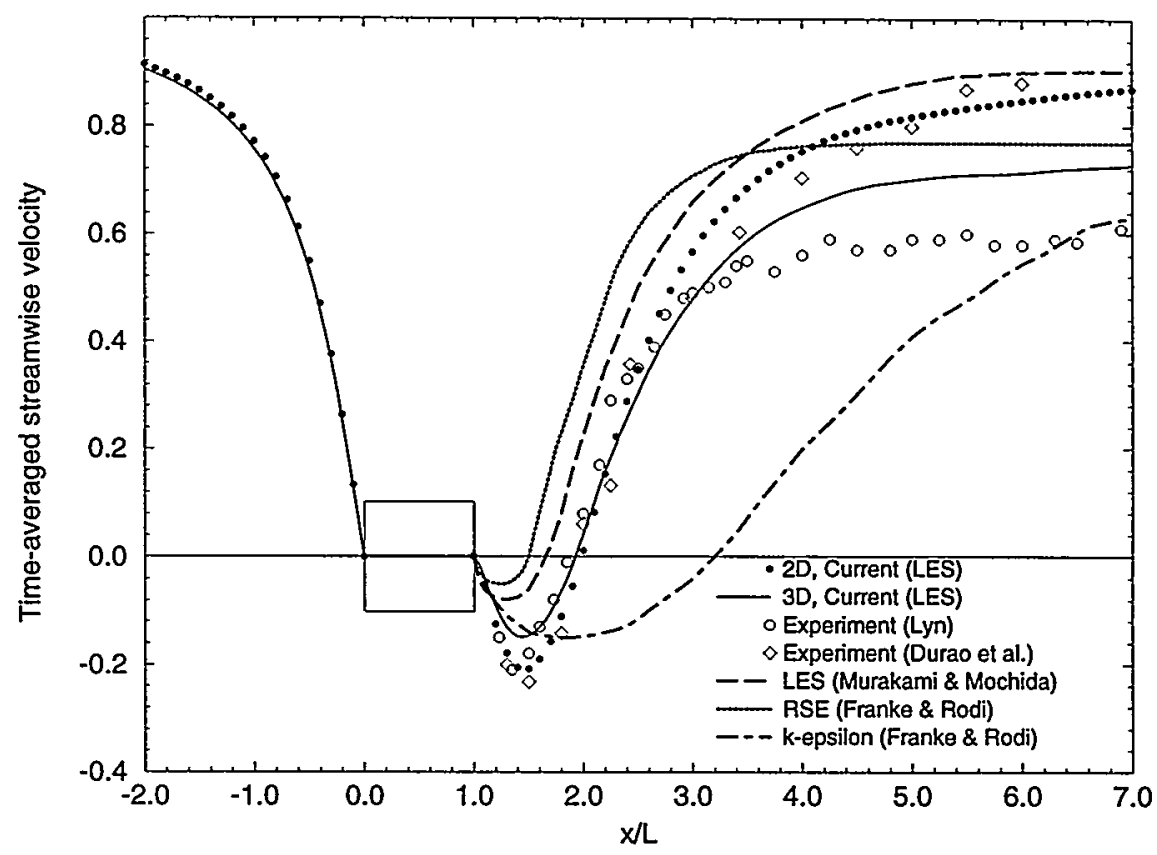

Figure 2. Time-averaged streamwise velocity.

\subsection{Flow Geometry}

The conducted numerical simulation is a flow around an infinitely long prism with a square cross section. Figure 1 is a schematic representation of the flow geometry. In the direction perpendicular to the surface of the paper, the prism is infinitely long, and a periodic boundary condition is applied.

The computational domain is $27 \mathrm{~L}$ in the streamwise direction, $18 \mathrm{~L}$ in the crossstream direction, and $2 L$ in the spanwise direction. The prism is located at the center in the lateral direction, and its front face is a distance of $8 L$ from the entrance flow boundary.

The computations were carried out primarily at the University of Notre Dame and at the National Center for Supercomputing Applications. A 3-D simulation at a Reynolds number of $10^{5}$ required $\approx 160 \mathrm{~h}$ of user time on an SGI Power Challenge computer, to march for a nondimensional time of 100 .

\subsection{Simulation Results of Velocity Field}

Time histories of the velocity and pressure field were generated by the abovedescribed method, with a computed potential flow for the same configuration or the output from a previous 2-D or 3-D simulation serving as the initial flow field. In each case, the vortex shedding process (for $R e=10^{5}$ ) begins without introducing any initial perturbation. When the 3-D simulation is initially started from a 2-D flow, it takes a nondimensional time of $\approx 50$ to reach a fully developed 3-D flow. The results reported hereafter are for the well-developed period. The statistical values (mean and RMS) were obtained over a time period of 500. Unless otherwise stated, all of the 


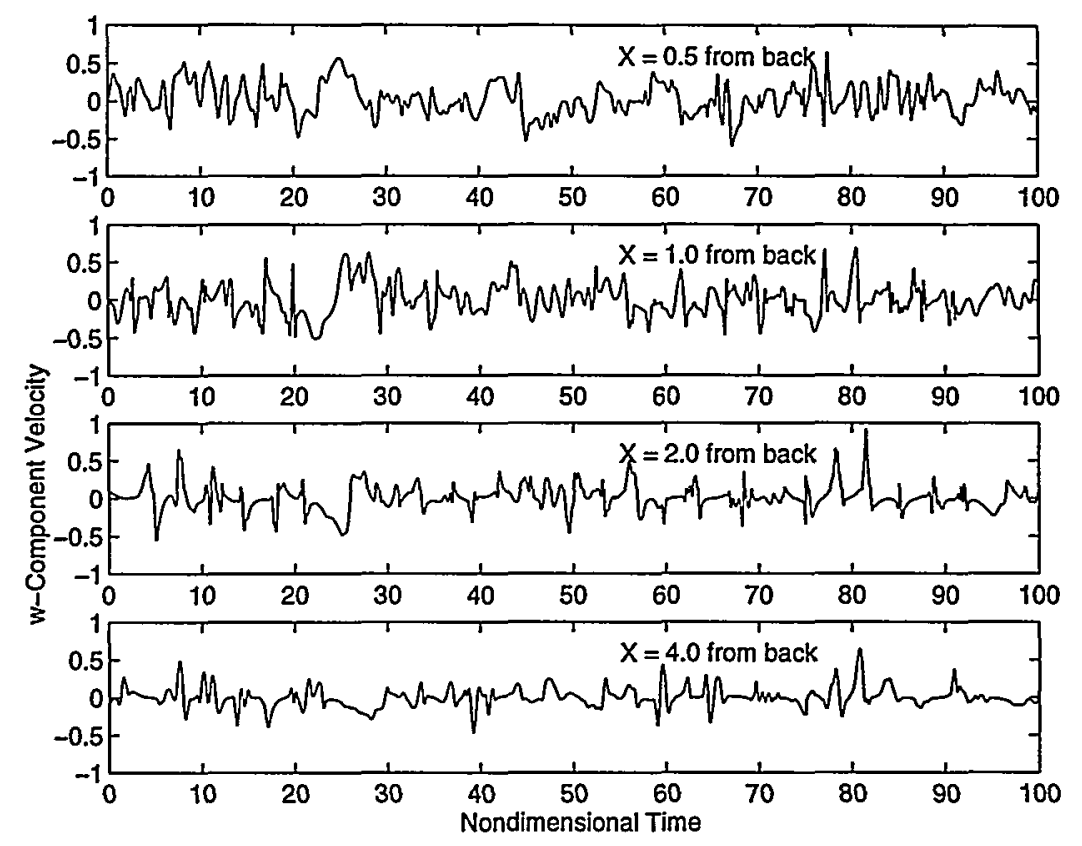

Figure 3. Time history of $w$-component velocity in wake.

reported 3-D results in this paper are based on the QUICK scheme, with a grid size of $D x=D y=0.05$ near the bluff body and a uniform $D z=0.2$ in the $z$-direction.

The time-averaged streamwise velocity on the symmetry line is reported in Fig. 2, together with the experimental results of Lyn (1989) and Durao et al. (1988), and the numerical results by Murakami and Mochida (1995), Franke and Rodi (1991) and Rodi (1993). Upstream of the square, the differences among the compared studies are extremely small. In the wake flow, both the current 2-D and 3-D simulation results show good agreement with experimental results. It should be noted that the 3-D results of Murakami and Mochida (1995) deviate from the current simulation results, although both are based on LES modeling. The discrepancy may be due to the numerical methods and boundary conditions that were employed rather than to the turbulence model itself. A similar discrepancy is also exhibited by the pressure results shown in the next subsection. Different turbulence models influence the results, as is demonstrated by the differences among the results obtained with $k-\epsilon$, Reynolds stress equation method (RSE) and LES in Fig. 2.

As seen in Fig. 2, the mean values from the 2-D simulation do not differ significantly from the 3-D results, although they were obtained with far less computational effort. Nevertheless, in the 3-D simulation, the spanwise component of velocity is present, and it redistributes energy from the $x-y$ plane. Figure 3 shows the time history of the spanwise velocity component at four downstream locations in the wake on the centerline, at distances of $0.5 L, 1.0 L, 2.0 L$, and $4.0 L$ from the rear face of the prism. 


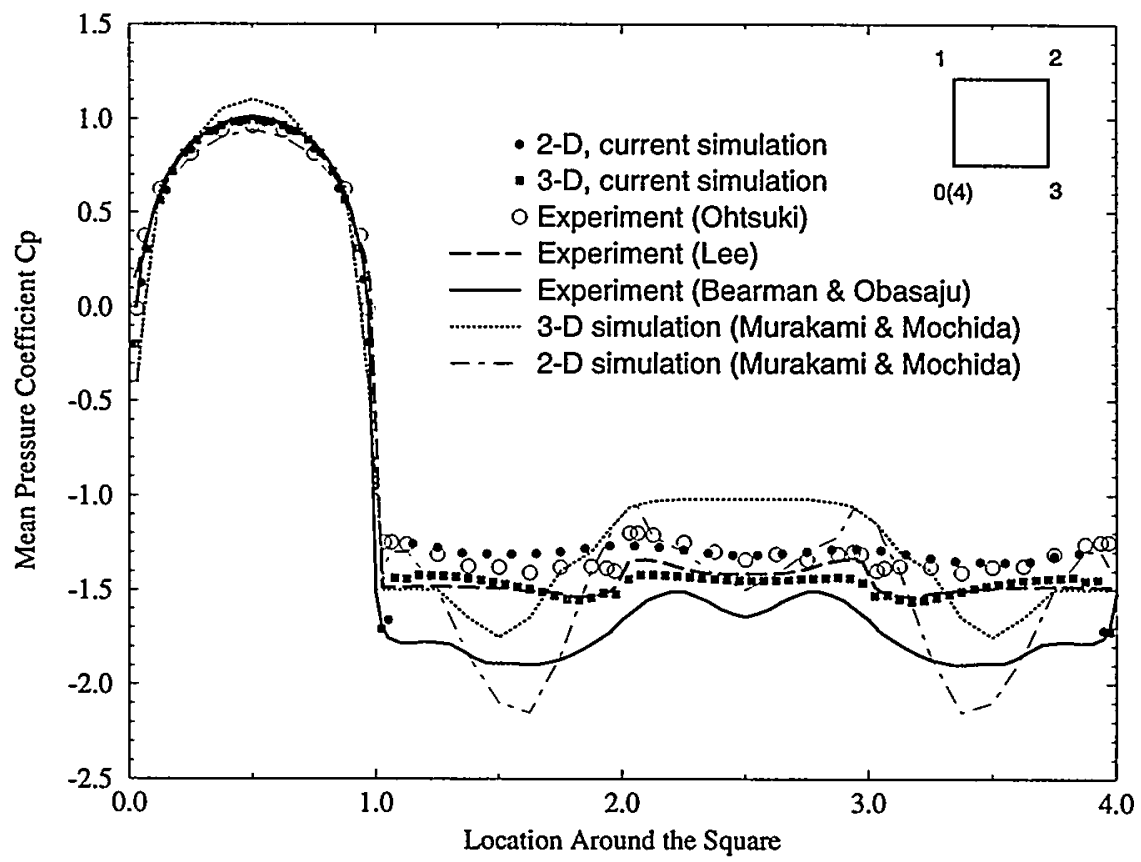

Figure 4. Distribution of mean pressure coefficient on surface of square prism

\subsection{Simulation Results of Pressure Field}

\subsubsection{Mean Pressure Distribution on Surface of Prism}

In Fig. 4, the distribution of the mean pressure coefficient $C_{p}=\bar{p} /\left(\frac{1}{2} \rho U_{0}^{2}\right)$ on the surface of a square prism is presented with the experimental data and numerical results of Murakami and Mochida (1995). The current 2-D and 3-D simulation results are generally close to one another and both compare well with the experimental data, except at two points at the front corners of the side faces. We emphasize that the grid size of the 3-D simulation is $D x=D y=0.05$ near the body, whereas for the 2-D simulation it is $D x=D y=0.1$. The 2-D results of Murakami and Mochida (1995) are very different from their 3-D simulation and experimental results. The 3-D results of Murakami and Mochida (1995) still exhibit a significant deviation from the experimental data in the regions of negative pressure. This is especially evident in the 3-D results for the leeward face, which show the pressure coefficient to be $\approx-1.02$, compared with the experimental value of near -1.3 to -1.5 . As mentioned earlier when discussing the results for the centerline velocity, the discrepancies between the current simulation and the results of Murakami and Mochida (1995) may not be due to the turbulence model, inasmuch as both used the LES, but may instead be a result of the numerical method or of boundary condition specifications. For example, the treatment of the outflow boundary by a free-boundary condition $(\partial / \partial n=0)$ may introduce errors in the simulated results. As noted earlier, a convective boundary condition is more realistic. 


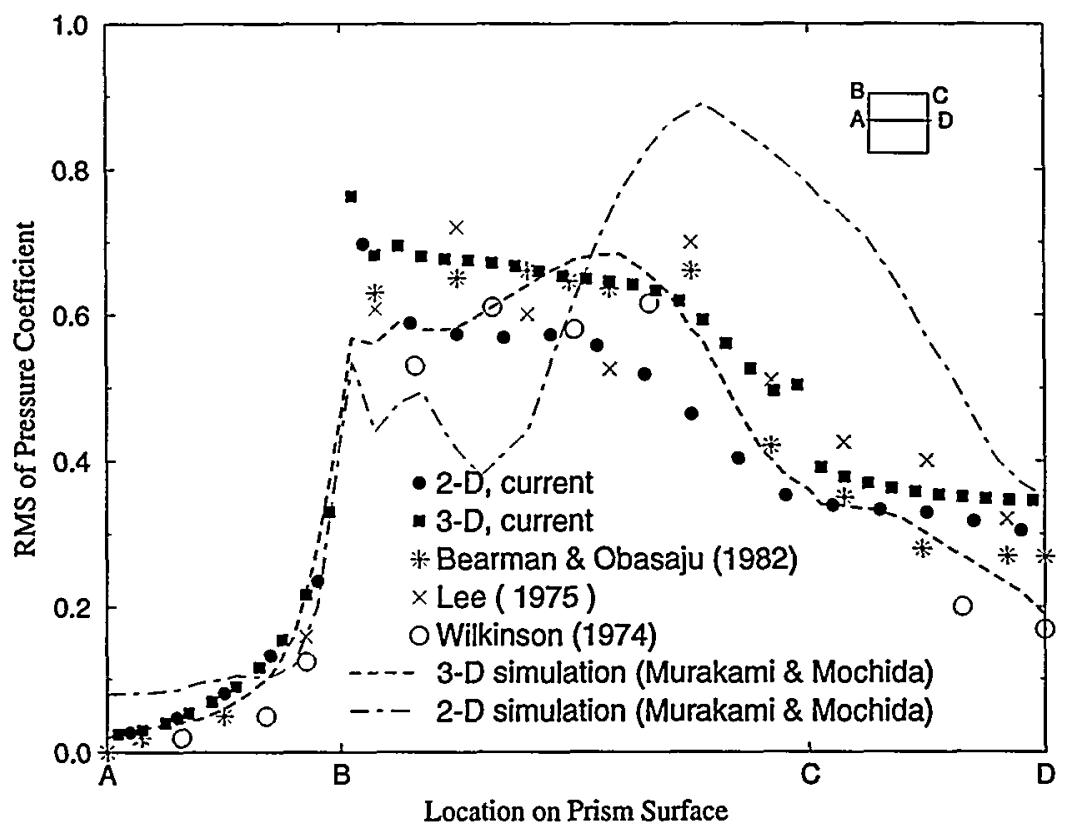

Figure 5. Distribution of RMS pressure.

\subsubsection{RMS Pressure Distribution on Surface of Prism}

In Fig. 5, we present RMS values of the pressure fluctuations on the surface of a square prism. The lowest RMS value is at the center of the front surface, whereas greater pressure fluctuations appear on the two side faces. On the back side, the fluctuations decrease as the center line on the back face is approached. Again, numerical results are presented with available experimental results and are found to be in a good agreement. The range of Reynolds numbers in the references cited in Fig. 5 are Bearman and Obasaju (1982), $R e=2 \times 10^{4}$; Lee (1975), $R e=1.76 \times 10^{5}$; and Wilkinson (1974), $R e=10^{4}-10^{5}$. Note that the 3-D results of Murakami and Mochida (1995) are also very close to the experimental results, whereas their 2-D results differ substantially.

\subsubsection{Integral Quantities}

Table 1 lists the calculated and experimental RMS values of $C_{L}$ and $C_{D}$, mean values of $C_{D}$, and Strouhal numbers. The 2-D and 3-D results (with near-body grid size of $1 / 20$ ) are close to one another and both agree well with experimental findings. At the same time, the 3-D results with a coarse grid size $(D x=D y=1 / 10)$ give much lower RMS values of both the lift coefficient $C_{L}$ and the drag coefficient $C_{D}$. The results with the grid size of $D x=D y=1 / 15$ are closer to the experimental values, whereas the results with the grid size of $D x=D y=1 / 20$ are the closest to the experimental values. The Strouhal numbers from simulations with differing grid sizes exhibit a similar trend. 
Table 1. Calculated and experimental integral quantities.

\begin{tabular}{lllll}
\hline Source & $\begin{array}{l}\text { RMS } \\
\text { of } C_{L}\end{array}$ & $\begin{array}{l}\text { Mean } \\
\text { of } C_{D}\end{array}$ & $\begin{array}{l}\text { RMS } \\
\text { of } C_{D}\end{array}$ & $\begin{array}{l}\text { Strouhal } \\
\text { Number }\end{array}$ \\
\hline 2-D, Dx,Dy =1/10 & 1.06 & 2.01 & 0.21 & 0.14 \\
3-D, Dx,Dy =1/20 & 1.15 & 2.14 & 0.25 & 0.135 \\
3-D, Dx,Dy =1/15 & 1.07 & 2.19 & 0.12 & 0.138 \\
3-D, Dx,Dy = 1/10 & 0.33 & 1.78 & 0.06 & 0.149 \\
Vickery (1966), 1 & 1.32 & & 0.17 & 0.12 \\
Vickery (1966), & 1.27 & & 0.17 & \\
Lee (1975) & 1.22 & 2.05 & 0.22 & \\
Bearman \& Obasaju (1982) & 1.2 & & & 0.13 \\
Nakamura \& Mizota (1975) & 1.0 & & & \\
Okajima (1982) & & & & 0.13 \\
\hline
\end{tabular}

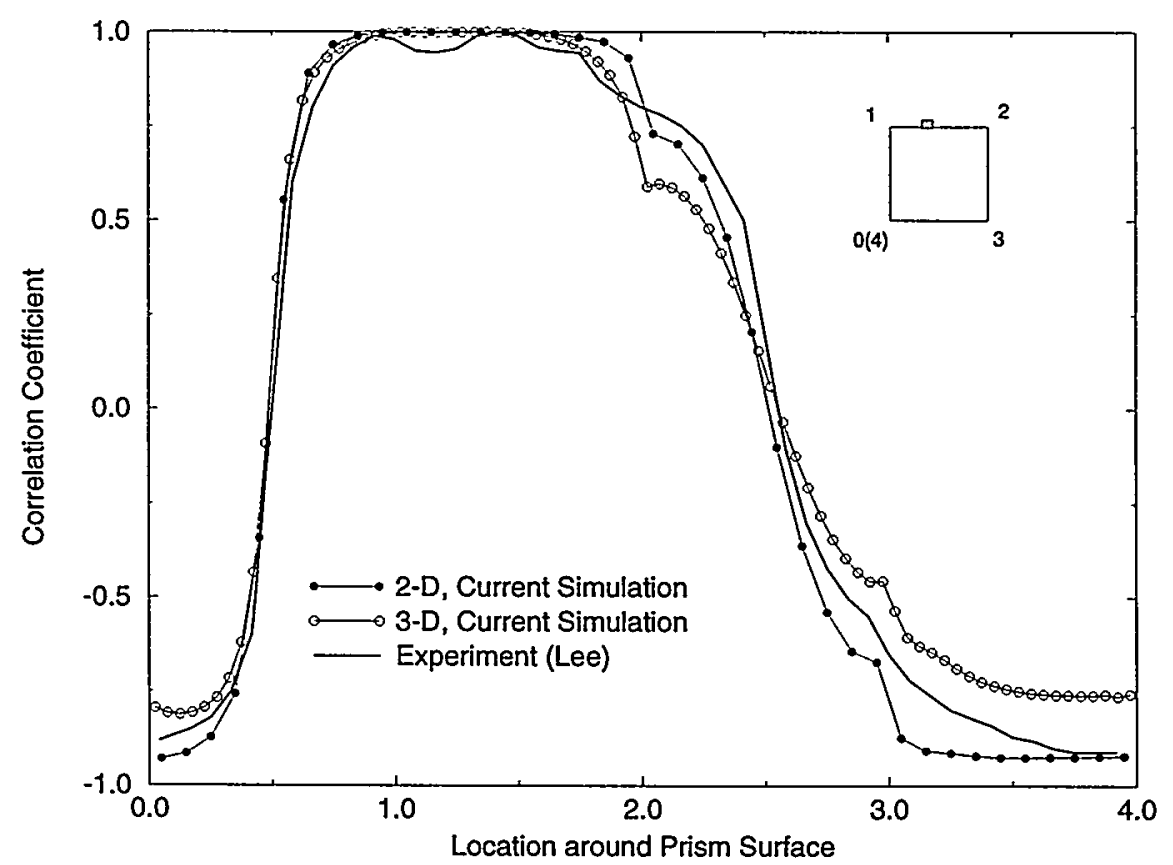

Figure 6. Chordwise correlation of pressure. 
Correlation of pressure fluctuations. Figure 6 shows the correlation coefficients of pressure fluctuations on the surface of the prism with reference to a point marked on the upper side face. Both the 2-D and 3-D results are plotted, as are the experimental results of Lee (1975). The results indicate a dominant presence of an antisymmetric correlation pattern associated with vortex shedding, and the numerical results are in very good agreement with experimental data. From Figs. 2, 4, 5, 6 and Table 1, it may be observed that the 2-D and 3-D results are not far apart, suggesting dominance of 2-D structure in the vortical flow field. This is in contrast with earlier reports by other investigators (e.g., Murakami and Mochida, 1995).

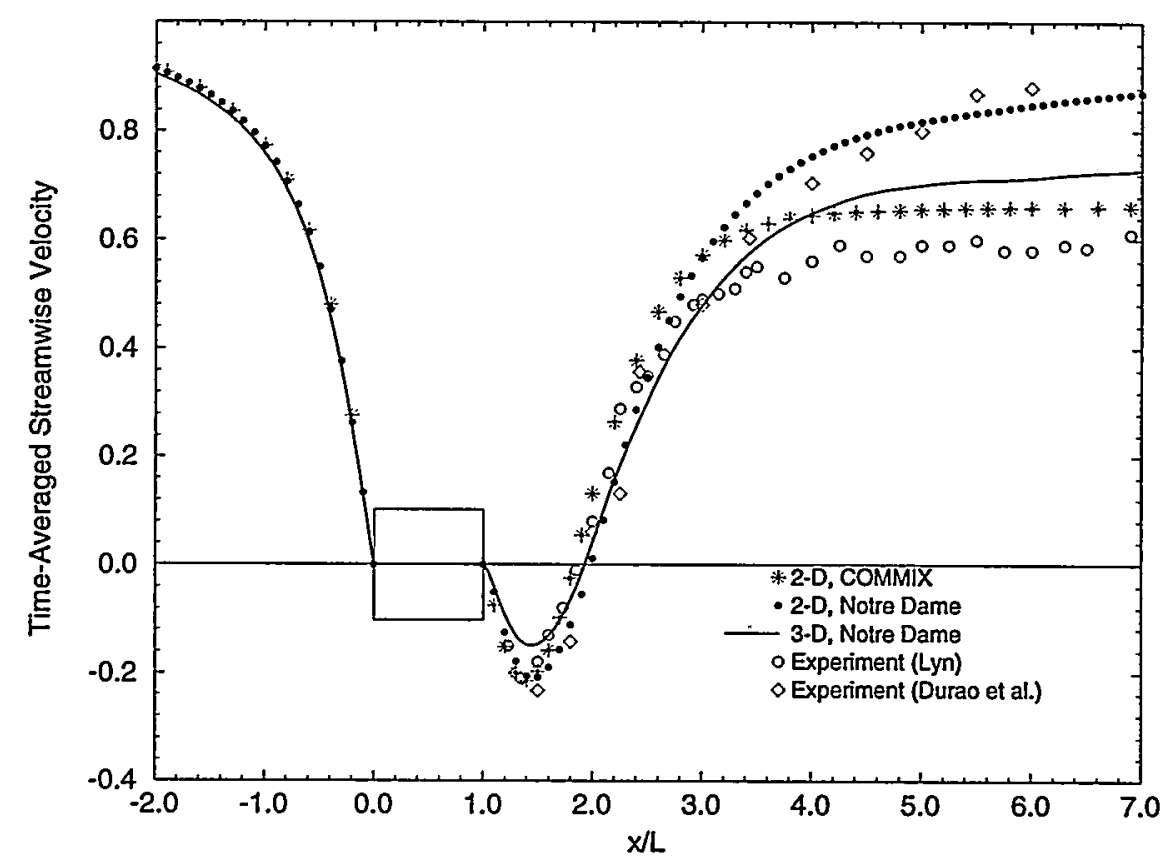

Figure 7. COMMIX results of time-averaged streamwise velocity.

\subsection{2-D Simulation Results from COMMIX}

To test the implemented LES model in the COMMIX code, a 2-D simulation of the flow described above was conducted. The results obtained from the COMMIX code are presented and compared with those obtained with the code developed by one of the authors at the University of Notre Dame.

Figure 7 shows the 2-D COMMIX results of the distribution of the time-averaged streamwise velocity along the centerline. It can be seen that all of the COMMIX and Notre Dame results predict the range of the reverse flow with high accuracy. The consistency is in contrast to the divergence demonstrated by the simulations presented in Fig. 2. All of the amplitudes of the maximum reverse flow velocity are also close to the two experimental data sets. The 3-D Notre Dame results slightly underpredict the reverse flow amplitude, but they are still in very good agreement with experimental data, especially when compared with numerical simulations of 
other authors, as depicted in Fig. 2. For the flow in the wake further downstream from the body, the two experimental sets of data deviate significantly, but the numerical results from both COMMIX and the Notre Dame code are still within the range of the experimental data.

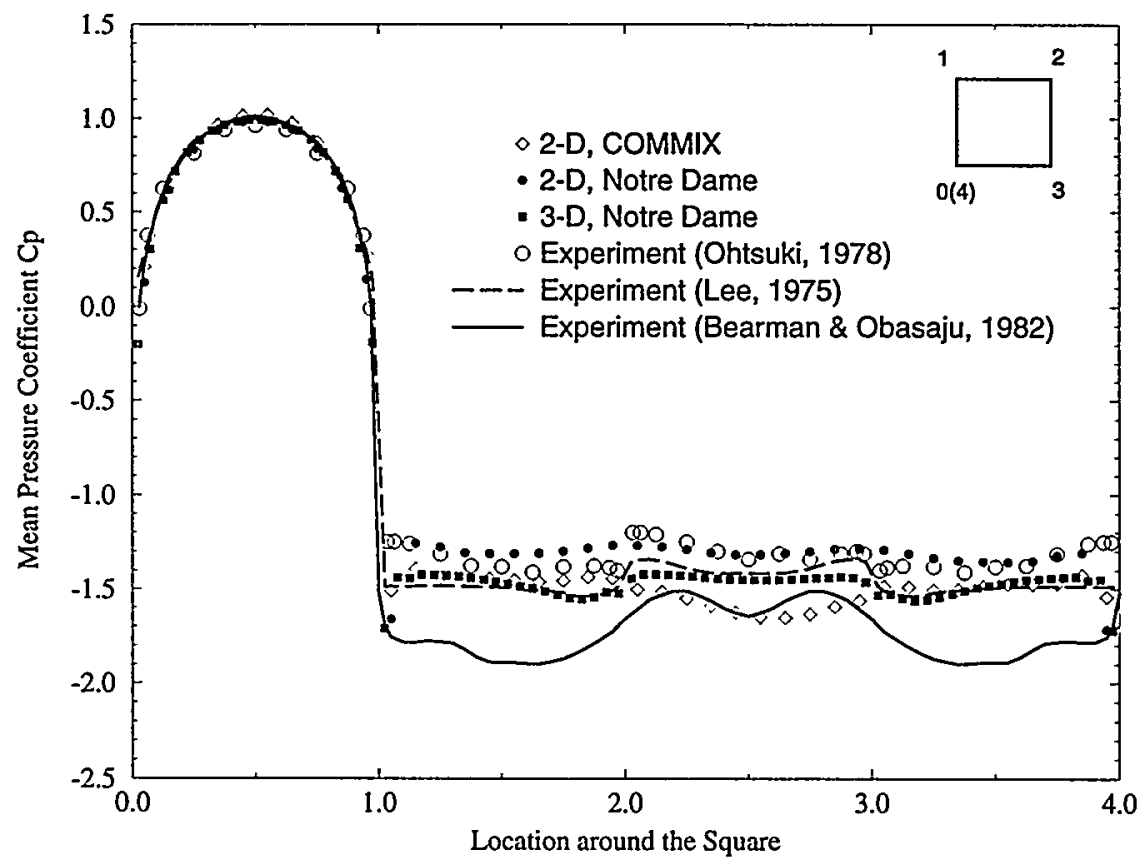

Figure 8. COMMIX results of distribution of mean pressure coefficient along surface of prism.

Figure 8 illustrates the distribution of the mean pressure coefficient along the surface of the prism. The COMMIX and Notre Dame results for the front and two lateral faces match closely, but they differ for the back face; however, both are still within the range of the experimental data sets. When we compare the results of Murakami and Mochida (1995), as presented in Fig. 4, with the COMMIX results, we observe that the COMMIX results do not show a high base pressure. The reason for this difference was not investigated further because of time limitations. It is understandable that results from different codes deviate from each other because of errors due to differing schemes and models, but the results presented here are still within the range of experimental data sets.

\section{Summary}

It has been experimentally confirmed that in a turbulent flow, large-scale eddies contain most of the energy and depend on the flow, whereas small-scale eddies are more isotropic and contain only a small part of the energy. Based on these facts, the large eddy simulation (LES) technique was derived by taking the spatial average of 
the Navier-Stokes equations. Thus, the large-scale fluid motions after filtering were resolved and computed explicitly and the small-scale motions were accounted for by a subgrid scale model. Application of the LES method to many flows has been reported in the literature and the results have been encouraging. The most widely used subgrid scale model was proposed by Smagorinsky (1966). A prominent recent improvement of the technique is the dynamic LES model, which derives the value of $C_{s}$ in the Smagorinsky model by using a test filter. In 1995, we implemented the LES method, based on the Smagorinsky model, in the ANL COMMIX code.

As an example of applications of the LES technique, results of a flow around a square prism are presented. Also included are results from simulations with a code developed by one of the authors at the University of Notre Dame, and some 2-D results from COMMIX. In the Notre Dame code, the QUICK scheme was used for the convection terms, and the Leith method was used for temporal marching. The subgrid scale viscosity was calculated using the Smagorinsky model. This 3-D numerical algorithm, based on a staggered grid, gives results that are in very good agreement with available experimental data, for both the velocity field and the pressure field of the flow. The 2-D results from the COMMIX code are in general agreement with the results obtained from the code developed at Notre Dame, and all of the results are within the range of the experimental data.

\section{Acknowledgments}

The authors thank Dr. Richard A. Valentin for his encouragement and support of this work.

\section{References}

[1] V. S. Arpaci and P. S. Larsen, 1974, Convective Heat Transfer, Prentice-Hall, Englewood Cliffs, NJ.

[2] P. W. Bearman and E. D. Obasaju, 1982, An experimental study of pressure fluctuations on fixed and oscillating square-section cylinders, J. Fluid Mech., 119, 297-321.

[3] J. P. Boris, F. F. Grinstein, E. S. Oran, and R. L. Kolbe, 1992, New insights into large eddy simulation, Fluid Dynamics Res., 10, 199-228.

[4] R. W. Davis and E. F. Moore, 1982, A numerical study of vortex shedding from rectangles, J. Fluid Mech., 116 , 475-506.

[5] R. W. Davis, E. F. Moore, and L. P. Purtell, 1984, A numerical-experimental study of confined flow around rectangular cylinders, Phys. Fluids, 27, 46-59.

[6] H. M. Domanus, Y. S. Cha, T. H. Chien, R. C. Schmitt, and W. T. Sha, 1990, COMMIX-1C: a three-dimensional transient single-phase computer program for 
thermal-hydraulic analysis of single and multicomponent engineering systems, NUREG/CR-5649, Argonne National Laboratory Report ANL-90-33.

[7] D. F. G. Durao, M. V. Heitor, and J. C. F. Pereira, 1988, Measurements of turbulent and periodic flows around a square cross-section cylinder, Exp. Fluids, 6, 298-304.

[8] R. Franke and W. Rodi, 1991, Calculation of vortex shedding past a square cylinder with various turbulence models, in Proc. 8th Symp. on Turbulent Shear Flows, p.189.

[9] M. Germano, U. Piomelli, P. Moin, and W. H. Cabot, 1991, A dynamic subgridscale eddy viscosity model, Phys. Fluids A, 3 (7), 1760-1765.

[10] F. H. Harlow and J. E. Welch, 1965, Numerical calculation of time-dependent viscous incompressible flow of fluid with free surface, Phys. Fluids, 8, 2182-2189.

[11] B. E. Launder and D. B. Spalding, 1972, Lectures in Mathematical Models of Turbulence, Academic Press.

[12] B. E. Lee, 1975, The effect of turbulence on the surface pressure field of a square prism, J. Fluid Mech., 69, 263-282.

[13] B. P. Leonard, 1979, A stable and accurate convective modelling procedure based on quadratic upstream interpolation, Computer Methods Appl. Mech. Eng., 19, 59-98.

[14] M. Lesieur and O. Metais, 1996, New trends in large-eddy simulations of turbulence, Ann. Rev. Fluid Mech., 28, 45-82.

[15] D. K. Lilly, 1967, The representation of small-scale turbulence in numerical simulation experiments, In Proc. IBM Scientific Computing Symp. on Environmental Sciences, IBM Form No. 320-1951, Thomas J. Watson Research Center, Yorktown Heights, 195-210.

[16] D. A. Lyn, 1989, Phase-averaged turbulence measurements in the separated shear flow around square cylinder, Proc. 23rd Cong. Int. Assn. Hydraulic Research, Ottawa, Ontario, Aug. 21-25, 1989, A85-A92.

[17] P. J. Mason, 1989, Large eddy simulation of turbulent shear flows, in Turbulent Shear Flows, von Karman Institute, Rhode Saint Genese, Belgium, LS 1989-03.

[18] P. J. Mason, 1994, Large-eddy simulation: A critical review of the technique, Q. J. R. Meteorol. Soc., 120, 1-26.

[19] S. Murakami and A. Mochida, 1995, On turbulent vortex shedding flow past 2-D square cylinder predicted by CFD, J. Wind Eng. Ind. Aerodyn., 54/55, 191-211.

[20] Y. Nakamura and T. Mizota, 1975, Unsteady lifts and wakes of oscillating rectangular prisms. Proc. A.S.C.E.: J. Eng. Mech. Div., 101 (EM6), 855-871. 
[21] Y. Ohtsuki, 1978, Wind tunnel experiments on aerodynamic forces and pressure distributions of rectangular cylinders in a uniform flow, Proc. 5th Symp. on Wind Effects on Structures, Tokyo, 169-175.

[22] A. Okajima, 1982, Strouhal numbers of rectangular cylinders, J. Fluid Mech., 123, 379-398.

[23] W. Rodi, 1993, On the simulation of turbulent flow past bluff bodies, J. Wind Eng. Ind. Aerodyn., 46/47, 3-19.

[24] T. S. Smagorinsky, 1963, General circulation experiment with primitive equations: Part I, basic experiments, Monthly Weather Rev., 91, 99-164.

[25] B. J. Vickery, 1966, Fluctuating lift and drag on a long cylinder of square crosssection in a smooth and in a turbulent stream, J. Fluid Mech., 125, 481-494.

[26] R. H. Wilkinson, 1974, On the vortex-induced loading on long bluff cylinders. Ph.D. thesis, Faculty of Engineering, University of Bristol, England.

[27] D. Yu and A. Kareem, 1996, Numerical Simulation of Pressure Field Around Two-Dimensional Rectangular Prisms, Technical Report No. NDCE-96-002, Department of Civil Engineering and Geological Sciences, University of Notre Dame, Notre Dame, IN. 
Internal:
F. C. Chang
R. B. Poeppel
R. A. Valentin
T. H. Chien
A. C. Raptis
R. W. Weeks
H. M. Domanus
R. C. Schmitt
TIS Files
W. A. Ellingson
W. T. Sha
C. A. Malefyt
J. G. Sun (5)

\section{External:}

DOE-OSTI (2)

ANL Libraries:

ANL-E

ANL-W

Energy Technology Division Review Committee:

H. K. Birnbaum, University of Illinois, Urbana

S.-N. Liu, Fremont, CA

I-W. Chen, University of Pennsylvania, Philadelphia

H. S. Rosenbaum, Fremont, CA

S. L. Sass, Cornell University, Ithaca, NY

R. K. Shah, General Motors Corp., Lockport, NY

S. Smialowska, Ohio State University, Columbus

Da-Hai Yu, State University of New York, Stony Brook 